\title{
¿De qué otra forma analizar el Proyecto Mesoamérica? Reflexiones desde la economía política internacional británica
}

\author{
Michelle Ruiz Valdes* \\ Sergio Vázquez Meneley ${ }^{* *}$
}

\section{RESUMEN}

El término Mesoamérica emergió en las relaciones regionales gracias a diferentes estrategias de cooperación entre México, Centroamérica, Colombia y República Dominicana que han derivado en lo que hoy se conoce como el Proyecto de Integración y Desarrollo de Mesoamérica. Sin embargo, a diez años de su creación, la mayor parte de los estudios al respecto son descriptivos y emplean un enfoque institucional que no resalta otros aspectos relevantes, como la inclusión de actores no gubernamentales y su participación en este programa de desarrollo regional. Con el propósito de hacer un primer acercamiento a estos aspectos dentro del Proyecto Mesoamérica, en este trabajo se busca explorar el marco teórico de economía política internacional (EPI) de procedencia británica, con los fines de identificar aquellos elementos que permiten ir más allá de los análisis tradicionales y profundizar, a partir de ellos, en el conocimiento del mencionado programa.

Palabras clave: Mesoamérica, Proyecto Mesoamérica, economía política internacional, cooperación regional, actores sociales.

* Maestría en cooperación internacional para el desarrollo. Asesora independiente (México). [michrv9228@gmail. com], [https://orcid.org/0000-0002-3524-422X].

** Estudiante de doctorado (C) en ciencias políticas y sociales, con especialidad en relaciones internacionales. Universidad Nacional Autónoma de México. Máster iberoamericano en cooperación internacional para el desarrollo. Consultor para el Programa de las Naciones Unidas para el Desarrollo (Pnud). (México). [sergio.vazquez18@gmail. com], [https://orcid.org/0000-0003-4520-7200].

Recibido: 10 de junio de 2019 / Modificado: 16 de julio de 2019 / Aceptado: 19 de julio de 2019

Para citar este artículo:

Ruiz Valdes, M. y Vázquez Meneley, S. (2020). ¿De qué otra forma analizar el Proyecto Mesoamérica? Reflexiones desde la economía política internacional británica. OASIS, 31, pp. 31-50

DOI: https://doi.org/10.18601/16577558.n31.04 


\section{How else to analyze the Meso- america Project? Reflections from the British International Political Economy}

\section{ABSTRACT}

Mesoamerica emerged in regional relations thanks to different cooperation strategies between Mexico, Central America, Colombia and the Dominican Republic that have resulted in what is known today as the Mesoamerica Integration and Development Project. However, ten years after its creation, most of the studies in this regard are descriptive and use an institutional approach that does not distinguish other relevant aspects such as the inclusion of nongovernmental actors and their participation in this regional program. In order to make a first approach to these aspects within the Mesoamerica Project, this paper proposes the use of the International Political Economy theoretical approach of British origin, in the interest of identifying those analytical elements that will go beyond the traditional approach and deepen our knowledge of this program.

Key words: Mesoamerica, Mesoamerica Project, International Political Economy, Social Stakeholders.

\section{INTRODUCCIÓN}

El Proyecto de Integración y Desarrollo de Mesoamérica cumplió diez años de existencia que se suman a los que ya tenía como Plan Puebla Panamá (PPP). Sin embargo, hasta el momento este programa de cooperación regional ${ }^{1}$ ha sido abordado académicamente mediante estudios históricos y económicos cuyos análisis se podrían ubicar dentro del marco teórico de la economía política internacional (EPI), de origen estadounidense, los cuales, principalmente, se concentran en un análisis gubernamental e institucional, dejando de lado estudios más profundos que abunden en las sinergias que se están conformando en la región a raíz de este programa, la articulación con actores sociales, así como su trabajo en red. Esto implicaría emplear otros enfoques dentro de la disciplina de Relaciones Internacionales, como la EPI de orientación británica, no obstante, valdría la pena cuestionar si su aplicación es factible o no para su estudio.

En este trabajo, se considera que el Proyecto Mesoamérica presenta algunas experiencias puntuales que podrían estudiarse empleando el enfoque de la EPI británica, ya que se registra una articulación de actores gubernamentales con otros actores sociales (fundaciones, academia, por mencionar algunos)

\footnotetext{
1 A pesar de que este mecanismo regional se denomina proyecto, es necesario distinguir, por su lógica de planificación, que corresponde a un programa. Por tal motivo, en este trabajo se hace referencia al Proyecto Mesoamérica como un programa de cooperación regional.
} 
en diferentes niveles de actuación y en distintos sectores temáticos dentro del Programa, tales como: salud, seguridad alimentaria, gestión de riesgo, etc. En este sentido, el objetivo de este artículo es identificar los rasgos de la EPI británica que son aplicables al caso del Proyecto Mesoamérica, esto se comprobará atendiendo la pregunta: ¡cuáles son los rasgos principales de la EPI británica que se podrían identificar dentro del mencionado programa de cooperación?

Para ello, el artículo se divide en dos apartados: en el primero, se realiza una revisión de dos de las principales escuelas de la EPI de acuerdo con Cohen (2011), así como sus ideas y elementos centrales; en el segundo apartado, se intentará identificar la manera en la que los elementos de la EPI británica se ven reflejados en el Proyecto Mesoamérica y se valorará hasta qué punto es factible emplear este enfoque; $y$, finalmente, en las conclusiones se delinean algunas ideas para un mejor entendimiento del programa. Cabe precisar que por la amplitud de este trabajo, el estudio está acotado a responder la pregunta de investigación sin profundizar en las particularidades regionales y nacionales en las que están insertos los países de la región, ello incluye, de igual manera, el tema de la cooperación para el desarrollo.

\section{EL PROYECTO MESOAMÉRICA ¿DESDE DÓNDE ESTUDIARLO?}

Desde su creación en 2008, el Proyecto de Integración y Desarrollo de Mesoamérica (en adelante, Proyecto Mesoamérica) se definió como una iniciativa regional "para implementar proyectos que resulten en beneficios concretos para las sociedades en materia de crecimiento económico, interconectividad y desarrollo social" (Amexcid, 2018) en torno a un nuevo término: Mesoamérica ${ }^{2}$, conformado por: Belice, Colombia, Costa Rica, El Salvador, Guatemala, Honduras, México, Nicaragua, Panamá y República Dominicana. Siendo esta una de las primeras veces que el término Mesoamérica se emplearía para hacer referencia a un programa de cooperación de alcance regional. Aunque, Almaguer-Kalisto (2016) menciona que la referencia a Mesoamérica ya se había registrado por parte de México, desde la creación del Plan Puebla Panamá (PPP) que es el antecedente inmediato del Proyecto Mesoamérica.

A la fecha, se cuenta con diferentes estudios que analizan las causas históricas por las que surgió esta iniciativa, su estructura institucional, así como sus principales resultados en materia de integración económica y, principal-

\footnotetext{
2 De acuerdo con especialistas, con Mesoamérica se hace alusión a una región con "prácticas agrícolas étnicas y similares, sistemas comerciales y político-religiosos, sistemas sociopolíticos complejos, redes de interacción a larga distancia y sistemas tecnológicos avanzados (Boone y Mignolo, 1994; Florescano, 1994)” (Citado por Almaguer-Kalisto, 2016, p. 10).
}

OASIS, ISSN: 1657-7558, E-ISSN: 2346-2132, N³1, Enero-Junio de 2020, pp. 31-50 
mente, en infraestructura energética y comunicaciones (Alizal, 2014; Espinoza y Augusto, 2010; Fromm, 2018a, 2018b; Sandoval, 2014; Morales, 2013; Toussaint y Garzón, 2017). No obstante, hay escasas referencias que tengan como objeto de estudio el componente social del Proyecto Mesoamérica y, por lo tanto, se orienten a analizar aspectos como: las sinergias sociales que se están conformando en la región a raíz de este programa, la participación de actores sociales, así como su articulación con actores gubernamentales en diferentes niveles y el trabajo en red.

Esto podría ser factible de analizar mediante el empleo del marco teórico de la economía política internacional (EPI), el cual ha sido utilizado para analizar los procesos de integración latinoamericanos y, no específicamente, para observar programas regionales de cooperación, como es el caso del Proyecto Mesoamérica.

De acuerdo con Cohen (2011), es posible dividir a la EPI en dos grandes escuelas: la norteamericana y la británica ${ }^{3}$. Siguiendo esta misma línea, Lombardo (2014) sostiene que ambos enfoques contienen rasgos convergen- tes en la forma en como visualizan el mundo, ya que los dos incluyen en el análisis tanto los aspectos políticos como económicos y, de igual manera, observan una continua y dinámica relación entre lo doméstico, lo regional e internacional.

Mas lo que marca la diferencia entre ambas es que cada una enfatiza en diferentes aspectos para observar el orden en el mundo. En el enfoque norteamericano predominan las aportaciones procedentes del realismo, el liberalismo y el neo institucionalismo internacional dentro de la disciplina de las Relaciones Internacionales y, por lo tanto, su orientación se dirige al análisis de los actores estatales, así como a sus motivaciones para cooperar con otros países con el fin de obtener beneficios tanto propios como compartidos.

Desde este enfoque la preocupación principal es la búsqueda de las motivaciones por crear instituciones que ayuden a solventar conflictos y a fungir como mecanismos reguladores entre los países ${ }^{4}$. Vinculado con ello, Vivares y Dolcetti (2016) identifican cuatro rasgos característicos para estudiar los regionalismos en América Latina desde la EPI norteamericana 5 .

3 Aunque ello varía dependiente a los autores, por su parte Tussie (2013) identifica cuatro: latinoamericana, estadounidense, británica y crítica.

4 Desde esta óptica, las instituciones son las que dan el curso a la cooperación regional, ya que son entes reguladores de la gobernanza al facilitar, supervisar y vigilar acuerdos pactados entre los países (Malamud, 2011). De acuerdo con Peters (1999), las instituciones contienen cuatro rasgos distintivos: el legalismo, el formalismo, el historicismo y el elemento normativo (Rodríguez y Ochoa, 2014).

5 La fijación de este modelo, de acuerdo con Vivares y Dolcetti (2016), se debe a que los estudios de la integración regional surgen a mediados del siglo pasado, precisamente cuando empezaba a conformarse la comunidad europea; sin embargo, los autores se distancian de este enfoque, al remarcar que los proyectos de regionalización en América Latina nacieron mucho antes de este período por lo que no es idóneo analizarlos desde una lógica europea y, además, porque las características de ambas regiones son totalmente distintas. 
Primeramente, resaltan la necesidad de crear instancias supranacionales, implicando la cesión de soberanía nacional por parte de los Estados miembros para dotar a estas de las atribuciones para la toma de decisiones y la ejecución de políticas sobre determinados aspectos regionales, a la usanza de la integración de la Unión Europea.

En segundo lugar, se prioriza el aspecto económico de la integración, considerando a este un proceso que se alcanza a través de una serie de etapas bien delimitadas que son resultado de una trayectoria histórica; por ende, el tema del desarrollo se vincula estrechamente con la actividad comercial y, en específico, con la apertura del mercado. Aunado a ello, el tercer rasgo asume la convergencia de los actores a través de instituciones, subestimando el poder desigual y las condiciones asimétricas existentes entre estos, dejando al libre mercado la regulación y la solución de los desequilibrios.

Por último, el cuarto rasgo plantea estrategias universales, bajo supuestos racionalistas y cientificistas, dejando de lado las particularidades generadas por diversos actores - principalmente los no gubernamentales-, los cuales participan en los procesos de desarrollo en diferentes ámbitos pero, puntualmente, en el local, formando parte de los procesos de regionalización que son fundamentales para la integración de la región.
La EPI estadounidense ha sido el enfoque preponderante en el análisis de los regionalismos latinoamericanos, incluyendo a los denominados regionalismos "abiertos" que proliferaron en la última década del siglo $\mathrm{xx}$, los que de acuerdo con Briceño (2014), fueron procesos que compartían -con sus respectivos matices- un modelo de integración basado en la apertura comercial e inserción internacional, vinculados con el modelo económico capitalista y de corte neoliberal liderado por los Estados Unidos; como el Mercado Común del Sur (Mercosur), el Tratado de Libre Comercio de América del Norte (TLCAN) sumándose a los ya existentes: el Mercado Común Centroamericano (MCCA) y la Comunidad Andina de Naciones (CAN) (Morales, 2013) ${ }^{6}$.

En reacción a la EPI norteamericana, surge la EPI de procedencia británica o también denominada pluralista, la cual sostiene que la integración es resultado de complejas interrelaciones que se presentan entre diferentes actores a través del tiempo, teniendo en cuenta que estas no solo se construyen por la actuación gubernamental sino que se articulan a través de redes formales e informales en distintos niveles de actuación así como variadas temáticas.

El enfoque pluralista procede de las aportaciones realizadas por autores formados en la tradición neorrealista en combinación con la teoría crítica -de línea neo gramsciana- así

\footnotetext{
6 Se denomina regionalismo abierto a las estrategias de integración que colocaban la atención en los procesos de liberalización económica de los países de la región latinoamericana (Morales, 2007). Desde una perspectiva crítica, Ojeda (2010) enfatiza que el modelo de regionalismo abierto pretendió imponer un orden político en las relaciones económicas internacionales y fue visto como la forma de obtener posibilidades para mantenerse en los mercados y beneficiar a las economías nacionales y regionales.
} 
como del materialismo histórico, por lo que no responde a un cuerpo organizado de teorías sino todo lo contrario, a una mixtura de ellas (Cohen, 2011; Vivares et al., 2013; Vivares y Dolcetti, 2016). El acento principal se pone en los procesos histórico-sociales que originaron los mecanismos de integración, reconociendo que los Estados ya no son los únicos actores sino que la regionalización comienza a articularse a través de otros agentes, principalmente, de procedencia no gubernamental.

Algunos autores consideran que esta EPI hace referencia a una multiplicidad de aproximaciones que tienen como denominador la crítica a los enfoques economicistas e institucionales (EPI norteamericana), y a la demanda por una valoración de lo político en los estudios de los regionalismos enmarcados en un contexto histórico y geográfico específico (Ayllón, 2016).

Para abundar en la EPI pluralista, se retomarán los cuatro rasgos mencionados en la EPI norteamericana y contrastar así ambas escuelas. En principio, si bien se reconoce la importancia de las instituciones, no necesariamente se remarca que estas tengan que ser entes supranacionales, planteando la construcción de otro tipo de estructuras en las que se privi- legie la configuración de relaciones informales entre una variedad de actores y temáticas, sin tener como modelo a seguir o referencia a la Unión Europea.

Dentro del segundo rasgo, desde la EPI británica se amplía el espectro para superar los temas comerciales y abarcar los sociales logrando una articulación de recursos y capacidades de los actores involucrados. En este sentido, han surgido algunos aportes de la EPI que estudian los nuevos regionalismos latinoamericanos en una lógica posneoliberal ${ }^{7}$ y poshegemónica ${ }^{8}$, que buscan transformar la estructura del sistema internacional y ubican al modelo neoliberal o al hegemón -específicamente a EE.UU.- como la causa de la perpetuación de las desigualdades entre países (Perrota, 2018; Perrota y Porcelli, 2018).

En el tercer rasgo, la EPI británica considera que la convergencia de actores no solo se da a través de vínculos institucionales de arriba hacia abajo, es decir, liderados por actores gubernamentales, sino que reconoce el valor de las interacciones que se producen por una variedad de actores que van de abajo hacia arriba; resaltando las diferentes capacidades que tienen los actores $y$, por ende, las asimetrías existentes entre ellos.

\footnotetext{
7 Sobre el regionalismo posneoliberal, Ojeda (2010) señala que estos procesos emergieron por múltiples causas, algunas de ellas relacionadas con el crecimiento de las economías de los países sudamericanos y la llegada a estos gobiernos de partidos políticos de izquierda con amplios márgenes electorales que les permitieron conducir sus acciones exteriores con mayor autonomía respecto a la registrada en períodos anteriores.

8 Este tipo de regionalismo se pronuncia en contra de las interferencias por parte de los EE.UU. como la potencia mundial y, sobre todo, de la expansión de su modelo neoliberal que se intentó trasladar a otros países a través de diferentes estrategias como la del Consenso de Washington (Briceño, 2014; Calvento y Rolandi, 2015; Lo Brutto y González, 2015; Ojeda, 2010).
} 
Por consiguiente, el contexto es un aspecto fundamental para este enfoque, ello se constata en el cuarto rasgo, pues la EPI británica no asume premisas únicas y homogéneas, sino que promueve esquemas más flexibles en los cuales tengan cabida procesos locales articulados por una gama de actores sociales. Ello permitiría incluir saberes propios que abonan a la definición de estrategias de desarrollo en los diferentes contextos.

Tomando en cuenta este marco, una región se representará por una configuración de relaciones históricas construidas a través del tiempo mediante procesos políticos y económicos, los cuales, a diferencia de la EPI norteamericana, se construirán de abajo hacia arriba, es decir, no son detonados por los gobiernos centrales de los Estados sino que se generan de facto por las interacciones tanto formales como informales, entre otros actores. Como señala Perrota: "la construcción de la región como un proceso ascendente, el papel de los actores no estatales y la afirmación de que son construcciones sociales. Por lo tanto, las regiones se encuentran en tensión a partir de proyectos políticos que disputan sobre la definición de lo que la región representa. Así, las ideas e identidades, tanto como los intereses, juegan un papel significativo en la definición y creación de una región" (2018, p. 16).

La tensión a la que refiere Perrota (2018), hace más fácil la distinción entre regionalismo y regionalización, entendiendo al primero, de acuerdo con Ayllón (2016), como las ideas y los proyectos políticos de región, de fuerzas formales e informales, que se generan de arriba a abajo con una orientación a la toma de decisiones en diferentes áreas de cooperación; mientras que la regionalización es un proceso subyacente de formación de regiones, mediante los nexos entre fuerzas sociales, individuales, colectivas, entre otros actores no estatales, que va de abajo hacia arriba. De acuerdo con Perrota (2018), ambos procesos son necesarios para que se produzca la integración, sin embargo, muchas veces estos no están en la misma sintonía.

Hasta este momento, se ha señalado que la EPI británica ha servido para estudiar los regionalismos, específicamente en Latinoamérica. Este enfoque cobró importancia con la conformación de la Alianza Bolivariana de los Pueblos de Nuestra América (Alba), la Unión de Naciones Sudamericanas (Unasur) y la Comunidad de Estados Latinoamericanos y Caribeños (Celac), en la primera década de este siglo, los cuales han sido estudiados desde este marco y pueden clasificarse como regionalismos posneoliberales o poshegemónicos? .

Dentro de estos procesos, la cooperación para el desarrollo se ha colocado como un instrumento fundamental a través del cual los países del Sur podían generar y mantener vínculos con otros países con los que se compartían lazos históricos y culturales y, además, registraban condiciones similares de desarrollo

9 La connotación de cada uno es distinta, como lo explican Perrota y Porcelli (2018), ambos sirvieron para adjetivar los estudios de regionalismos en América Latina en la primera década del siglo xxI. Ahondando en las características de ellos, pero no necesariamente en sus orígenes y, principalmente, en la orientación de la integración que se pretendía seguir.

OASIS, ISSN: 1657-7558, E-ISSN: 2346-2132, N³1, Enero-Junio de 2020, pp. 31-50 
originadas por las condiciones asimétricas de la estructura internacional.

Las acciones de cooperación para el desarrollo que empezaron a incrementarse con creces dentro de estos mecanismos, se insertaron en la modalidad denominada Cooperación Sur-Sur (css), es decir,

aquella cooperación que otorgan unos países medianamente desarrollados a otros de similar o menor desarrollo relativo en las áreas o sectores en las que han logrado éxitos o han adquirido una experiencia propia, y que se pueden extender mediante diferentes mecanismos de intercambio (técnicos, económicos, científicos, etc.) para contribuir al desarrollo de capacidades en otros países (Ojeda, 2010, p. 93).

Dentro de estos procesos regionales la css se convirtió en un instrumento fundamentalmente político, el cual, de acuerdo con Surasky (2016), tuvo el propósito de privilegiar el intercambio de saberes producidos entre países del Sur ${ }^{10}$, así como la intención de romper con los vínculos de dependencia característicos del modelo neoliberal.

Además de la cooperación, dentro de la EPI británica también se le otorga un peso sustantivo a la identidad, la cual se concibe como "un sustento imprescindible en la construcción y consolidación de las regiones" esta identidad se conformará, primordialmente, mediante las interacciones de diferente índole entre múltiples actores no gubernamentales, dentro de las cuales subyacen ideas, culturas y creencias que pueden ser entendidas como ejes de acción, reacción o contestación política (Vivares y Dolcetti, 2016).

Respecto a la ideología, Briceño (2014) resalta la importancia de los actores no estatales, señalando que estos fungen como fuerzas "regionalizadoras", las cuales se organizan en una especie de defensa frente a los embates del mercado, como parte de un segundo movimiento que se valdría del "regionalismo para detener la expansión de las esferas no mercantiles de la vida humana (Polanyi, 2003)" (Citado en Briceño, 2014, p. 27).

Dentro de los procesos sudamericanos, la css fue un instrumento que impulsó el regionalismo y la integración, abonando en la construcción de una identidad común. Lo que no necesariamente se observó en otros mecanismos regionales, como la Alianza del Pacífico, la CAN, el Mercosur y el MCCA, así como el Sistema de Integración Centroamericana (Sica), en los que sí se presentó la css, pero con otras características, ya que estos procesos dieron continuidad al regionalismo "abierto" sin criticar ni plantear rupturas con el modelo liberal ${ }^{11}$.

\footnotetext{
10 Sobre la definición de Sur, Surasky señala que con este término se entiende a una "posición política que sostiene la necesidad de recuperar aquello que ha sido ocultado por la modernidad y el orden internacional subsiguiente de bases europeo-occidentales y capitalista, buscando establecer un orden internacional que refleje las diferencias" (2016, p. 57).

11 Lo que Romano (2012) califica de contradictorio, ya que por una parte promovía un discurso basado en la integración regional mediante la cooperación sin contemplar una ruptura con el sistema sino una perpetuación de estos lazos de dependencia.
} 
Por lo tanto, en este contexto se puede identificar lo que algunos autores denominan como Latinoamérica dividida en cuanto a los regionalismos, ya que se mantuvieron modelos “abiertos" liderados por gobiernos - de derecha y centro derecha-, junto a los regionalismos contra hegemónicos o posneoliberales antes mencionados (Ayllón, 2016; Surasky, 2016; Vivares y Dolcetti, 2016). De esta forma, en el siglo Xxi no se puede hablar de un modelo único de integración en la región sino de la existencia de una heterogeneidad o fragmentación de los mismos (Briceño, 2014) e incluso en la forma de analizarse como lo enfatizan Perrota y Porcelli (2018).

El Proyecto Mesoamérica surge dentro de este contexto de fragmentación, pero siguiendo marcadamente un modelo del regionalismo "abierto", pues aunque este es un programa de cooperación regional, tanto en su nombre como en sus objetivos se hace hincapié en la búsqueda de la integración y el desarrollo de Mesoamérica, lo que se refrendó en la transición del ppp al Proyecto Mesoamérica con la inclusión de Colombia y República Dominicana para configurar a esta como una región o subregión en el ámbito de las relaciones internacionales contemporáneas.

A partir de esta fecha, para Colombia y México, como los principales donantes, el Proyecto Mesoamérica es visto como una de sus principales acciones de css que va dirigida a la región geográfica en donde tienen mayor influencia: Centroamérica. Aunque muchas veces estas acciones se solapan con las que ejecuta el Sica, como el proceso de integración más consolidado y que tiene agendas muy similares a las del programa analizado en este trabajo. Sin embargo, desde que inició se ha intentado que el Proyecto Mesoamérica se convierta en una estrategia regional y no se perciba como una acción unilateral del gobierno mexicano.

Algunos estudios señalan que los resultados del Proyecto Mesoamérica están orientados a la integración física, concentrándose en la creación de "infraestructura para favorecer el libre tránsito y abrir las puertas a los capitales privados" (Toussaint y Garzón, 2017, p. 17), beneficiando únicamente a ciertos grupos involucrados en sectores como energía, transporte e infraestructura, y relegando proyectos de desarrollo social de envergadura con los que se logre un impacto sustantivo en el bienestar de las sociedades mesoamericanas. Otros argumentan que el Proyecto Mesoamérica es una estrategia de influencia e integración con los EE.UU. a través de tratados comerciales y acuerdos de seguridad que se han firmado en las últimas décadas y que en múltiples formas vinculan a estos países con la potencia continental, y en este cometido México funge como un importante aliado y pivote regional (Orozco, 2014; Sandoval, 2014).

Con lo hasta ahora expuesto, se distingue que el Proyecto Mesoamérica cuenta con los elementos para estudiarse dentro de la EPI norteamericana; sin embargo, el objetivo de esta investigación es ir más allá del enfoque tradicional e identificar aquellos elementos que desde la EPI británica podrían contribuir a complementar y profundizar los estudios sobre este programa; para ello se hará una revisión de los rasgos revisados de esta escuela en el siguiente apartado. 


\section{2. ¿ES POSIBLE ANALIZAR AL PROYECTO MESOAMÉRICA DESDE LA EPI BRITÁNICA?}

Como se adelantó, el Proyecto Mesoamérica es un mecanismo regional latinoamericano que tiene su antecedente en el PpP ${ }^{12}$. Desde su creación, en 2008, se inserta dentro del Mecanismo de Diálogo y Concertación de Tuxtla, el cual constituye el foro mesoamericano al más alto nivel para que, entre otras cosas, se analicen y acuerden los asuntos de interés común y se impulsen acciones regionales en el marco de la Agenda Mesoamericana de Cooperación, instrumento que busca la articulación de iniciativas y la complementariedad de actores en el área económica y social.

Para coordinar los proyectos y acciones de cooperación dentro de la mencionada agenda, el Proyecto Mesoamérica cuenta con una estructura que favorece el diálogo entre los países miembros; está compuesta por diferentes entidades intergubernamentales con atribuciones para gestionar iniciativas con instancias nacionales en la mayoría de los casos y en otros, sin embargo, se ha buscado la articulación con otros actores no gubernamentales, como se verá más adelante. Mas esta estructura no tiene una naturaleza supranacional, su organización se vincularía más a una instancia flexible que favorece la configuración de relaciones en di- ferentes niveles, situación que va en línea con el primer rasgo de la EPI británica.

En la figura 1 se presenta la estructura del Proyecto Mesoamérica.

Como se aprecia en la figura 1, las interacciones que se dan en el Proyecto Mesoamérica son en un nivel técnico, operativo y financiero para planificar y coordinar acciones con instancias gubernamentales, como las secretarías o ministerios de los países miembros, así como con organismos del Sistema de Naciones Unidas, la banca de desarrollo y las organizaciones regionales de integración, puntualmente, dentro del Sica; pero se observan escasos espacios que contemplen el involucramiento de otro tipo de actores, como lo pudieran ser la academia, las organizaciones de la sociedad civil, las fundaciones y los grupos comunitarios.

Para observar lo anterior, a continuación, se muestra una tabla que contiene información de las cumbres del Mecanismo de Tuxtla, dentro de esta revisión se resalta el interés y el reconocimiento expresado a otros agentes del desarrollo, pero también se confirma la naturaleza estatal del Proyecto Mesoamérica y la participación técnica y financiera de organismos intergubernamentales.

A través de las declaraciones es posible distinguir la importancia que se le ha dado a la configuración de relaciones con la iniciati-

12 El ppp fue una estrategia de integración promovida por México a principios del siglo xxI y acogida por los países centroamericanos en el marco de la Cumbre Extraordinaria del Mecanismo de Diálogo y Concertación de Tuxtla realizada en 2001 en la ciudad de San Salvador, El Salvador (Altmann y Beirute, 2007; Espinoza y Augusto, 2010). De acuerdo con la Declaración Conjunta, el ppp se concebía como "un instrumento impulsor de desarrollo y la integración regionales y con objeto de profundizar y fortalecer el entendimiento político y la cooperación internacional” (2001, p. 1). 
Figura 1

Estructura del Proyecto Mesoamérica

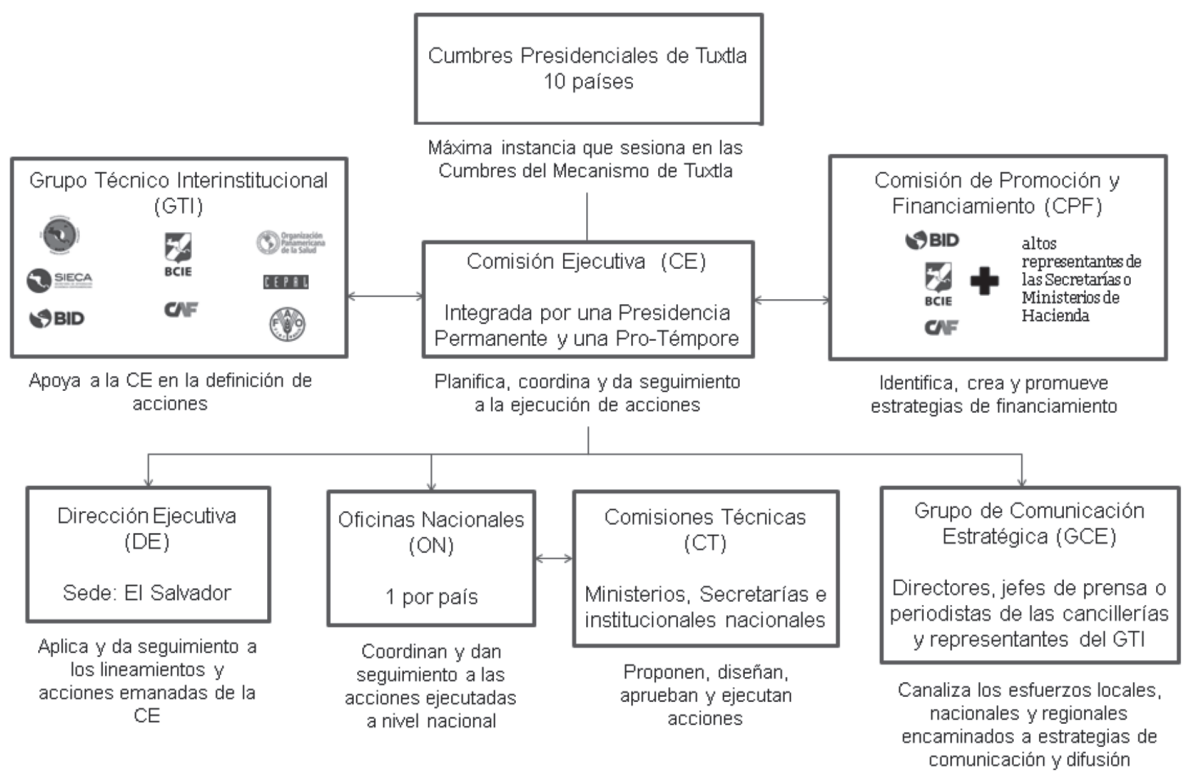

Fuente: Retomado y adaptado de Proyecto Mesoamérica.

va privada a través de asociaciones públicoprivadas, modelos multisectoriales y esquemas de trabajo conjunto donde se incluyan a las pequeñas y medianas empresas; lo que refleja una preponderancia de los actores gubernamentales y la inclusión casi exclusiva de actores privados, y en pocas excepciones se plantea la participación de actores sociales.

Un ejemplo de esto último, se presentó con la inclusión de la academia en la creación del Instituto Mesoamericano de Salud Pública (IMSP), el cual se consolidó gracias a la colaboración con instancias académicas de México, Centroamérica y Colombia. En este sentido, la academia aparece como un actor relevante para generar y fortalecer capacidades técnicas a nivel regional.

Como se observa en las declaraciones, hay un énfasis en la inclusión del sector privado en lo económico y una mención limitada de los actores sociales como agentes fundamentales para contribuir a los beneficios de la región y crear procesos colectivos de desarrollo; lo que refrenda que en el Proyecto Mesoamérica prevalece una visión gubernamental de alto nivel. Ello, no solo se evidencia en la estructura y en las declaraciones del Proyecto Mesoamérica, sino también en los consensos construidos sobre determinados conceptos, como el de cooperación. 
Tabla 1

Cumbres del Mecanismo de Diálogo y Concertación de Tuxtla 2008-2017

\begin{tabular}{|c|c|}
\hline Cumbres & Aspectos abordados \\
\hline $\begin{array}{l}\text { Declaración de } \\
\text { Villahermosa } \\
2008\end{array}$ & $\begin{array}{l}\text { - Búsqueda conjunta de fuentes de financiamiento complementarias y otras modalidades de la coo- } \\
\text { peración. } \\
\text { - Aplicar esquemas de asociación público-privada. } \\
\text { - Reconocimiento de los actores que han apoyado, técnica y financieramente la iniciativa mesoa- } \\
\text { mericana: miembros del GTI, organismos regionales, agencias de cooperación públicas y privadas y } \\
\text { gobiernos de terceros países. }\end{array}$ \\
\hline $\begin{array}{l}\text { Declaración de } \\
\text { Guanacaste } \\
2009\end{array}$ & $\begin{array}{l}\text { - Reconocimiento de los actores que han apoyado, técnica y financieramente, a la iniciativa mesoame- } \\
\text { ricana, en particular a los organismos internacionales que conforman el GTI. } \\
\text { - Reconocimiento de los ministerios de salud y de las instancias académicas de México, Centroamérica } \\
\text { y Colombia para contribuir a la constitución del Instituto Mesoamericano de Salud Pública (IMSP). } \\
\text { - Promover la realización de programas, proyectos y actividades específicas que contribuyan a una mayor } \\
\text { participación de las pequeñas y medianas empresas en el comercio exterior en la región. }\end{array}$ \\
\hline $\begin{array}{l}\text { Declaración de } \\
\text { Cartagena } \\
2010\end{array}$ & $\begin{array}{l}\text { - Reafirmar el compromiso fundamental que implica para los países de Mesoamérica el cumplimiento } \\
\text { de los Objetivos del Desarrollo del Milenio (oDM), con una cooperación más eficaz, promoviendo y } \\
\text { fortaleciendo las alianzas para el desarrollo en el ámbito de la css. } \\
\text { - Reconocimiento del lanzamiento de la asociación público-privada en temas de salud: Iniciativa Salud } \\
\text { Mesoamérica } 2015 \text {. } \\
\text { - Continuar el fortalecimiento de los mecanismos de coordinación del Proyecto Mesoamérica con } \\
\text { otros foros regionales y multilaterales, mediante un mayor involucramiento de las autoridades de } \\
\text { cooperación de los países, particularmente en la formulación, monitoreo y evaluación de proyectos } \\
\text { de cooperación. }\end{array}$ \\
\hline $\begin{array}{l}\text { Declaración de } \\
\text { Mérida } \\
2011\end{array}$ & $\begin{array}{l}\text { - La cooperación para el desarrollo constituye una herramienta privilegiada o un vehículo pertinente } \\
\text { para contribuir al desarrollo sustentable de la región y para construir mecanismos de colaboración } \\
\text { que coadyuven a la consecución de los oDM. } \\
\text { - Reconocimiento del rol desempeñado por el sector privado en el fortalecimiento de las relaciones } \\
\text { económicas de la región mediante acciones que han permitido compartir sus experiencias en la ma- } \\
\text { teria. }\end{array}$ \\
\hline $\begin{array}{l}\text { Declaración de } \\
\text { Antigua } \\
2015\end{array}$ & $\begin{array}{l}\text { - Impulsar proyectos económicos conjuntos y acordar acciones de cooperación regional, en todos los } \\
\text { ámbitos establecidos como prioritarios en el contexto del mecanismo, en aras del desarrollo sostenible } \\
\text { del área. }\end{array}$ \\
\hline $\begin{array}{l}\text { Declaración de } \\
\text { San José } \\
2017\end{array}$ & $\begin{array}{l}\text { - Los compromisos globales establecidos en la Agenda } 2030 \text { para el Desarrollo Sostenible implican la } \\
\text { promoción de acciones conjuntas bajo una visión colaborativa, a través de esquemas multisecto- } \\
\text { riales y las alianzas público-privadas para contribuir a la consecución de los Objetivos de Desarrollo } \\
\text { Sostenible (oDs). } \\
\text { - Reconocimiento del papel que desempeña el sector privado en la generación de oportunidades para } \\
\text { el desarrollo de la población, así como el potencial de su contribución a los gobiernos en la búsqueda } \\
\text { de acercamientos intrarregionales y multisectoriales. } \\
\text { - Reconocimiento de la importancia de las alianzas público-privadas como herramientas privilegiadas } \\
\text { para impulsar la cooperación y el desarrollo. }\end{array}$ \\
\hline
\end{tabular}

Fuente: Elaboración propia con base en las Declaraciones de las Cumbres de Tuxtla. No fue posible acceder a los documentos de las Cumbres de 2014 y 2019. 
Para el Proyecto Mesoamérica, la css regional es entendida como la "colaboración que ofrece un país al conjunto de otros países miembros y que aporta buenas prácticas a una o más líneas de trabajo que conforman la Agenda Mesoamericana de Cooperación" (Proyecto Mesoamérica, 2017, p. 2). Como se evidencia, se reconoce el valor que aportan los países al intercambiar sus buenas prácticas, las cuales son conceptualizadas como "un saber y un saber hacer" que ha desarrollado una institución nacional para solucionar cualquier desafío del desarrollo y que cuenta con logros comprobados.

De acuerdo con ello, al menos discursivamente se ha introducido la importancia de rescatar e intercambiar los saberes de cada país con sus contrapartes mesoamericanas; mas estos saberes siguen quedándose a un nivel gubernamental sin contemplar los conocimientos y prácticas de actores sociales imprescindibles para cualquier acción que persiga objetivos de desarrollo.

En lo que respecta al segundo rasgo de la EPI británica, se distingue que la transición del ppp al Proyecto Mesoamérica supuso una ampliación en la agenda de trabajo y un cambio en la manera de gestionar las acciones. Mientras que la primera iniciativa estuvo enfocada en la integración física (construcción de plataformas de infraestructura eléctrica, telecomunicaciones y transporte), en la segunda, desde el discurso se puso el acento en temas sociales, tales como salud, medio ambiente, gestión de riesgos y vivienda (Proyecto Mesoamérica, 2018).

Con ello se intentó no solo atender temas económicos sino ir ampliando la oferta programática hacia lo social para la promo- ción gradual del desarrollo de las sociedades mesoamericanas; no obstante, a diez años de estos cambios prevalece una continuidad en la lógica de regionalismo abierto en donde los temas económicos tienen un peso importante, como lo demuestran los principales resultados de los estudios sobre este programa (Espinoza y Augusto, 2010; Toussaint y Garzón, 2017).

Como parte de la orientación temática, la Agenda Mesoamericana de Cooperación comprende en el eje económico, los sectores de telecomunicaciones, energía, facilitación comercial y competitividad, y transporte y, en el eje social, se encuentran los sectores de salud, medio ambiente, gestión del riesgo, vivienda, y seguridad alimentaria y nutricional. Para dar cuenta de la orientación de la agenda, se registra que la concentración en el eje económico no solo se presenta en el porcentaje de las acciones, sino también en la asignación de los recursos financieros, siendo el sector transporte al que se le destinaron más recursos entre 2008 y 2015 (Proyecto Mesoamérica, s.f.), relegando los temas sociales nodales para el desarrollo como la salud y la gestión de riesgo.

Considerando estos datos, el segundo rasgo de la EPI británica únicamente se presenta en el discurso del Proyecto Mesoamérica, así como en la definición de las áreas de actuación de la agenda, pero no en términos reales de implementación. A pesar de esta aseveración, es importante reconocer los esfuerzos incipientes que se están desarrollando en lo social para la convergencia de vínculos con actores no gubernamentales y la generación de sinergias que se conforman de abajo hacia arriba, lo que iría en línea con el tercer rasgo de la EPI británica. 


\section{Gráfico 1}

\section{Acciones concluidas y en ejecución de los ejes económico y social dentro del \\ Proyecto Mesoamérica así como sus montos para el período 2008-2015 (Porcentaje y monto total acumulado en mdd)}

\section{Concluidas}

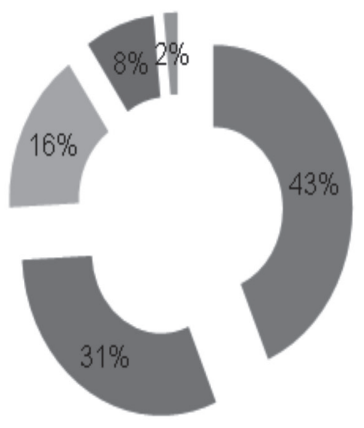

- Transporte $\$ 342.3 \mathrm{mdd}$

- Facilitación comercial y competitividad $\$ 5.2$ mdd

=Energía $\$ 262 \mathrm{mdd}$

- Telecomunicaciones $\$ 2.1 \mathrm{mdd}$

- Gestión del riesgo $\$ 0.8 \mathrm{mdd}$
Ejecución

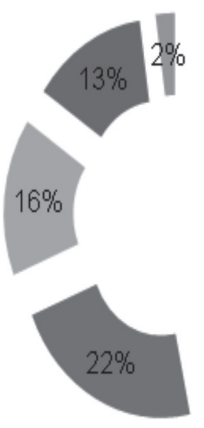

- Transporte $\$ 1,681.2 \mathrm{mdd}$

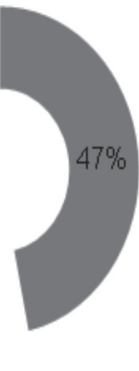

- Energía $\$ 553.9$ mdd

$=$ Salud $\$ 223.5 \mathrm{mdd}$

-Facilitación comercial y competitividad $\$ 5.75 \mathrm{mdd}$

- Telecomunicaciones $\$ 0.6$ mdd

Fuente: Retomado y adaptado de Proyecto Mesoamérica (s.f.).

Un ejemplo de ello podría ser la iniciativa Mesoamérica sin Hambre ${ }^{13}$ dentro del sector de seguridad alimentaria y nutricional del Proyecto Mesoamérica, que se caracteriza por ser una acción de Cooperación Sur-Sur Triangular $(\mathrm{Css}-\mathrm{T})^{14}$, la cual "se ejecuta [en Centroamérica, República Dominicana y Colombia], con el apoyo técnico de la Organización de las $\mathrm{Na}$ - ciones Unidas para la Alimentación y la Agricultura (FAO) y la cooperación financiera del Gobierno de México” (Adonay, 2018, p. 51).

Esta iniciativa se basa en un modelo de trabajo fundamentado en la gestión territorial a través de los gobiernos locales, la "apropiación por parte de los países involucrados a través de una planificación centrada en la demanda”

13 En el marco de xv Cumbre de Tuxtla (2015) se incorporó la Iniciativa Mesoamérica sin Hambre a la cartera del Proyecto Mesoamérica.

14 La CSs-T puede ser entendida como la "modalidad de Cooperación Sur-Sur en la que participan un conjunto de actores que, pudiendo todos ellos realizar distintos tipos de aportes (técnicos, financieros u otros) se reparten el ejercicio de tres roles: el de los así denominados primer oferente y receptor (uno o varios países en desarrollo, en cada caso), y el de segundo oferente (país en desarrollo, país desarrollado, organismos regional o multilateral, o alguna asociación de ellos). El rasgo oficial está determinado por el rol del primer oferente, quien actúa como principal responsable del fortalecimiento de capacidades" (Citado en Segib, 2014, p. 107). 
(Díaz, 2018, p. 13) y de mecanismos ad hoc que:

a) Busquen el establecimiento de vínculos de colaboración con otros actores (parlamentos, sociedad civil, academia, entidades gubernamentales y organizaciones locales de agricultura familiar);

b) Faciliten la adaptación a los contextos políticos y sociales de los países mesoamericanos; y

c) Favorezcan la movilización de recursos a través de estrategias novedosas de cofinanciamiento, las cuales pretenden que las municipalidades designen un porcentaje de sus presupuestos a la seguridad alimentaria y nutricional, respetando su autonomía (Adonay, 2018; Díaz, 2018).

$\mathrm{Al}$ menos en el discurso, estos mecanismos ad hoc se orientan a fomentar la articulación de otros actores -no solo gubernamentales- en materia de seguridad alimentaria, así como a buscar la complementariedad de estas acciones y los recursos que se destinan a ellas, lo que podría resultar inédito en estas acciones regionales, con la aplicación de estos mecanismos que supondrían un reconocimiento por el trabajo que realizan otros actores, así como por sus capacidades en esta temática.
Otra de las experiencias que se ha desarrollado en el marco del Proyecto Mesoamérica y que ha involucrado a otros actores, es el Programa Educativo de Apoyo a las Capacidades en Gestión de Riesgos y Adaptación al Cambio Climático en Centroamérica, el cual tuvo una duración aproximada de dos años, de octubre de 2016 a septiembre de 2018. Esta acción fue producto de la cooperación trilateral entre México, la Agencia Suiza para el Desarrollo y la Cooperación (Cosude, por sus siglas en inglés) y la DE del Proyecto Mesoamérica para fortalecer las capacidades del Consejo Superior Universitario Centroamericano (CSUCA). De acuerdo con la página del Proyecto Mesoamérica, su objetivo fue "contribuir a que los estudiantes egresados de las universidades miembros del csucA [contaran] con conocimientos y herramientas para integrar la gestión integral de riesgos ante desastres y la adaptación al cambio climático en los procesos de desarrollo de la región"15.

Si bien es cierto que en este programa se involucraron los gobiernos de México y Suiza, se incluyó a otros actores en los procesos de cooperación, como las universidades centroamericanas, debido a que el csuca es una organización que integra a los sistemas públicos de Educación Superior del Sica, para promover el desarrollo de las universidades a

15 Para mayor información consultar: "Programa Educativo de Apoyo al Desarrollo y Consolidación de Capacidades Técnicas e Institucionales en Gestión de Riesgos y Adaptación al Cambio Climático en Centroamérica”, Proyecto Mesoamérica, http://www.proyectomesoamerica.org/index.php/acerca-delpm/63-proyectos-ejecucion-depm/265programa-educativo-de-riesgos

OASIS, ISSN: 1657-7558, E-ISSN: 2346-2132, N³1, Enero-Junio de 2020, pp. 31-50 
través de la cooperación y el trabajo conjunto con la sociedad y el Estado (CSUCA, 2017).

Mas no es la primera vez que en el Proyecto Mesoamérica se involucra a la academia en los temas sociales, como ya se vio, se registran experiencias en la consolidación del IMSP; por lo que en materia de salud se aprecia un avance en la incorporación de actores que van más allá de los países y de los gobiernos al fomentar la participación de universidades, pero también de fundaciones (Fundación Carlos Slim y la Fundación Bill y Melinda Gates) a través de la Iniciativa Salud Mesoamérica 2015.

Considerando lo anterior, se observa que existen algunas experiencias que implican una articulación multinivel o por colaboración en red con actores sociales, esta funciona bajo modelos de cofinanciamiento y complementariedad entre un "sinnúmero de actores políticos y sociales, como autoridades gubernamentales en los tres niveles (municipal, estatal y federal), cuerpos legislativos, organizaciones de la sociedad civil, academia y sector privado, entre otros" (Ponce, 2016, p. 86), no obstante, estos casos son excepcionales y no un común denominador dentro del Proyecto Mesoamérica.

$\mathrm{Al}$ establecer una mayor conexión entre estos casos con el tercer rasgo de la EPI británica, se aprecia que no se cumple en su totalidad debido a que los proyectos se desprenden de la toma de decisiones que va de arriba hacia abajo, pues a pesar de que se ha intentado integrar a otros actores, persiste la necesidad de establecer una planificación centrada en la demanda de las sociedades mesoamericanas, así como de actores sociales y no solo gubernamentales o, bien, de la iniciativa privada. Ello implica tener en cuenta las demandas locales de tal manera que se incida en una toma de decisiones horizontal y no vertical o asistencialista dentro del Proyecto Mesoamérica.

El cuarto rasgo de la EPI británica se relaciona con la adaptación al contexto y a los procesos locales articulados por una gama multiactor, que estaría estrechamente ligado con el rasgo anterior y, como se observó, hay una incipiente inclusión de actores sociales, que únicamente se ha presentado en algunos sectores como el de salud, gestión de riesgo y seguridad alimentaria; por lo tanto, con la información pública que proporciona el Proyecto Mesoamérica es complicado conocer si los proyectos dentro de este programa tomen en cuenta las prioridades locales de desarrollo, se adapten al contexto e incluyan saberes de las comunidades.

A manera de conclusión, se observa que el Proyecto Mesoamérica contiene rasgos que pueden ser analizados desde la EPI estadounidense y la EPI británica, aunque más de la primera que de la segunda, pues este programa de cooperación está inserto en las relaciones económicas internacionales que vinculan a sus países miembros con los EE.UU. Sin embargo, se observan algunos rasgos que van en línea con la EPI británica como la creación de instituciones no necesariamente supranacionales $y$, en algunos sectores muy puntuales, se reconocen los aportes técnicos y financieros de otros actores, como la academia y las fundaciones.

Este primer acercamiento permite ver la pertinencia de analizar el Proyecto Mesoamérica desde otras perspectivas y profundizar en aspectos del mismo que no necesariamente han sido tomados en cuenta y que permitirán conocer los vacíos que tiene este programa, para 
construir una estrategia con una sólida orientación a la sostenibilidad y a la apropiación del desarrollo en diferentes niveles - no solo a nivel de los países-y en donde se produzca la incorporación de diferentes actores, principalmente, de los actores sociales.

\section{CONCLUSIONES}

El Proyecto Mesoamérica es un programa de cooperación regional que se inscribe dentro de un modelo de regionalismo abierto, característico de los procesos de integración que están en curso entre Norte y Centroamérica, y que se distinguen por su alta concentración en temas económicos, así como por una estructura institucional marcadamente intergubernamental en una lógica de actuación que va de arriba abajo, y que deja poco espacio para incluir actores no gubernamentales. No obstante, la diversidad temática de este programa ha sentado las bases para que, de manera incipiente y en algunos sectores específicos -como gestión del riesgo, salud y seguridad alimentaria-, comiencen a articularse redes de colaboración en distintos niveles de actuación e incluso con actores no gubernamentales como la iniciativa privada, academia y fundaciones.

Esto último da pie a que el Proyecto Mesoamérica sea factible de explorar y analizar desde otros enfoques no tradicionales en los que se pueda profundizar en los aspectos sociales del mismo; concretamente, desde la EPI británica, la cual se propone en este artículo y que cuenta con ciertos rasgos que se ven reflejados en el mencionado programa, a pesar de que se cuenta con escasa información que permita hacer un análisis a profundidad desde este enfoque. Mas ello no debe ser un obstáculo para investigar el componente social de este programa, así como las dinámicas que van en contra de las relaciones de poder existentes en la región y que plantean nuevas formas de cooperación e integración, resaltando los saberes que se producen en las sociedades, así como los rasgos propios culturales, sociales e históricos que subyacen en el origen de Mesoamérica.

Como balance de este trabajo, si bien la EPI británica es un enfoque útil, se tendría que contemplar la construcción de un enfoque híbrido entre las escuelas de la EPI que permita hacer un análisis económico y político ad hoc del Proyecto Mesoamérica, que tome en cuenta las particularidades del mismo; pues aún persisten importantes retos para incluir la dimensión social, cuya respuesta tendría que pasar por el diseño de mecanismos novedosos en los que se fomente "una agenda común, con objetivos, programas y métodos compartidos de trabajo" (Morales, 2014, p. 230), abonando así, a la articulación social de esta región.

\section{REFERENCIAS}

Adonay, F. (2018). Micsur, un modelo de gestión territorial en seguridad alimentaria y nutricional. En Proyecto Mesoamérica. La Agenda Mesoamericana de Cooperación para un Desarrollo Social Incluyente: Alcances e impactos en salud, vivienda, seguridad alimentaria y nutricional (pp. 44-57). El Salvador: Dirección Ejecutiva.

Alba, F. and Castillo, M. (2012). New Approaches to Migration Management in Mexico and Central America. Washington, DC: Migration Policy Institute.

Alizal A., L. (2014). La integración con el sur: México y Centroamérica en las primeras décadas del siglo 
xxI. En Soto, W. (Edit.), Política internacional e integración regional comparada en América Latina.

San José, Costa Rica: Flacso, unA, pp. 147-155. Almaguer-Kalixto, P. (2016). The Politics of Technical Assistance in Regional Integration Processes: Mesoamerican Insights. Iberoamerican Journal of Development Studies, 5, No. 1, pp. 5-30.

Altmann, B. y Beirute, T. (2007). Dossier: Plan Puebla Panamá. San José: Secretaría General de la Flacso. Ardilla, M. (2015). El regionalismo mexicano en la Alianza del Pacífico y el Proyecto Mesoamérica. En Carta Económica, vol. xxviI, No. 116, juliodiciembre, pp. 36-62.

Ayllón, B. (2016) ¿Latinoamérica dividida? Procesos de integración y cooperación Sur-Sur. Quito: Editorial Iaen.

Briceño, R. J. (2014). Del regionalismo abierto al regionalismo poshegemónico en América Latina. En Soto, W. (Edit.), Política internacional e integración regional comparada en América Latina. San José, Costa Rica: Flacso, unA, pp. 23-33.

Caldentey, P. (2014). El sistema de integración centroamericana y la cooperación Sur Sur. En Ayllón, B.; Ojeda, T. y Surasky, J. (2014). Regionalismose integración en América Latina. España: Catarata, pp. 108-127.

Calvento, M. y Rolandi, M. (2015). Reconfiguración de la Cooperación Sur-Sur en la región latinoamericana y la participación internacional de los actores subnacionales. En Revista Mexicana de Ciencias Politicas y Sociales, Unam, vol. Lx, No. 224, mayo-agosto, pp. 315- 347.

Cohen, B. (2011). Toward a new consensus: from denial to acceptance. In N. Phillips and C. Weaver (Eds.), International Political Economy: debating the past, present and future. London: Routledge, pp. 231-239.
Díaz, T. (2018). Mesoamérica sin hambre: Trabajando juntos por la erradicación del hambre y la pobreza rural en nuestra región. En Cuaderno GTI. Alianzas para una Cooperación Regional Eficaz: El Grupo Técnico Interinstitucional (GTI) como socios esenciales del Proyecto Mesoamérica (pp. 11-29). El Salvador: Dirección Ejecutiva.

Espinoza, B. y Augusto, G. (2010). Proyecto de Integración y Desarrollo de Mesoamérica (2008). En El Cotidiano, vol. xxv, No. 159, enero-febrero, 2010, pp. 59-68.

Fromm, L. (2018a). La Agenda Mesoamericana de Cooperación: ¿Con qué pinceles dibujamos la próxima década? En Proyecto Mesoamérica. El Proyecto de Integración y Desarrollo de Mesoamérica: Una reflexión sobre los alcances y resultados de una cooperación mesoamericana renovada (pp. 1938). El Salvador: Dirección Ejecutiva.

Fromm, L. (2018b). Hacia una región mejor preparada ante los fenómenos naturales. En Proyecto Mesoamérica. Transporte, Gestión de Riesgo, Facilitación Comercial y Transporte una visión intersectorial regional en Mesoamérica (pp. 49-58). El Salvador: Dirección Ejecutiva.

Ibarra, A.; Delgadillo, A.L. y Pérez, J. A. (2017). Caso de Estudio: Sistema Mesoamericano de Salud Pública del Proyecto de Integración y Desarrollo de Mesoamérica. Primera fase de operación 20102015. Ciudad de México: Oxfam.

Keohane, R. O. (1993). Institucionalismo neoliberal: Una perspectiva de la política mundial. En R.O. Keohane. Instituciones internacionales y poder estatal: ensayos sobre las teorias de las relaciones internacionales (pp. 13-38). Buenos Aires: Grupo Editor Latinoamericano.

Lombardo T., P. (2014). La Cooperación Sur-Sur: ¿̨nuevas formas de actuar y/o nuevas representaciones 
de poder? La cooperación brasileña, período 2008-2012. Tesina. Quito: IAEN.

Lo Brutto, G. y González R., E. (2015). Regionalismo estratégico, cooperación Sur-Sur y desarrollo en América Latina en el siglo xxi. En Bajo el Volcán, vol. 15, No. 22, marzo-agosto, Buap, pp. 151-176.

Malamud, A. (2011). Conceptos, teorías y debates sobre la integración regional. En Norteamérica, Año 6, No. 2, julio-diciembre, pp. 219-249.

Matute, J. (2018). El Sistema Mesoamericano de Salud Pública en el contexto de la agenda de salud global: logros y perspectivas. En Proyecto Mesoamérica. La Agenda Mesoamericana de Cooperación para un Desarrollo Social Incluyente: Alcances e impactos en salud, vivienda, seguridad alimentaria y nutricional (pp. 21-33). El Salvador: Dirección Ejecutiva.

Morales F., M. E. (2007). Un repaso a la regionalización y el regionalismo: Los primeros procesos de integración regional en América Latina. En Confines Relaciones Internacionales y Ciencia Política, vol. 3, No. 6, Monterrey, ago./dic.

Morales F., M. E. (2013). Unasur, Alba-TCP y Centroamérica ¿ejes de la integración regional o liderazgos individuales en América Latina? En Bocanegra G., C. y Vázquez, R. M. (2013). Procesos de integración económica regional. Ciudad de México: Pearson, pp. 84-105.

Morales, H. (2014). Integración y la agenda social en Mesoamérica: Poder y lucha política en la región. Guatemala: Movimiento Tzuk Kim-pop.

Ojeda, T. (2010). La Cooperación Sur-Sur y la regionalización en América Latina: el despertar del gigante dormido. En Relaciones Internacionales, No. 15, octubre. Geri-UAM, pp. 90-112.

Orozco C., M. (2014). La sombra estadounidense en las relaciones entre México y Centroamérica. En So- to, W. (Edit.), Política internacional e integración regional comparada en América Latina. San José, Costa Rica: Flacso, una, pp. 183-193.

Perrota, D. y Porcelli, E. (2018). El regionalismo es lo que la academia hace de él. En Revista Uruguaya de Ciencia Politica, No. 28 (1), pp. 183-218.

Perrota, D. (2018). El campo de estudios de la integración regional y su aporte a las relaciones internacionales, una mirada desde América Latina. En Relaciones Internacionales, No. 38. JunioSeptiembre, pp. 9-39.

Peters, G. (1999). El nuevo institucionalismo. Teoría institucional en ciencia politica, Barcelona: Gedisa.

Ponce, E. (2016). Fundamentos y actuación de la cooperación descentralizada y para el desarrollo local. En E. Ponce; S. Lucatello y R. Velázquez (Coords.), Teoría y práctica de la cooperación internacional para el desarrollo: Una perspectiva desde México, México: AMei/CGCID/REMECID, pp.75-89.

Proyecto Mesoamérica. (2018). Alianzas para una Cooperación Regional Eficaz: el Grupo Técnico Interinstitucional como socios esenciales del Proyecto Mesoamérica. Cuadernos Mesoamericanos, El Salvador: Proyecto Mesoamérica.

Proyecto Mesoamérica. (2018a). El Proyecto de Integración y Desarrollo de Mesoamérica: Una reflexión sobre los alcances y resultados de una cooperación mesoamericana renovada, El Salvador: Dirección Ejecutiva. Rodríguez, S. P. y Ochoa, B. L. (2014). Los regionalismos en el siglo xxi: conceptualización, retos y perspectivas en la nueva estructura internacional. Revista Enfoques, vol. XII, No. 20, pp. 77-100.

Romano, S. (2012). La inversión extranjera, las translatinas y la acumulación en el Mercosur. Revista del Centro Cultural de la Cooperación, enero-agosto, 
14/15. Consultado en http://www.centrocultural. coop/ revista/articulo/324/

Sandoval, P. J. (2014). El proyecto de desarrollo e integración de Mesoamérica en el marco de la estrategia de seguridad nacional de Estados Unidos. En Soto, W. (Edit.), Politica internacional e integración regional comparada en América Latina, San José, Costa Rica: Flacso, unA, pp. 157-182.

Surasky, J. (2016). Apuntes para una conceptualización del Sur desde la integración y la cooperación SurSur. En Ayllón, B., ¿Latinoamérica dividida? Procesos de integración y cooperación Sur-Sur. Quito: Editorial Iaen, pp. 51-74.

Toussaint, M. y Garzón, M. (2017). El Proyecto Mesoamérica: ¿Éxito o fracaso? Límites de la cooperación de México hacia Centroamérica. En Entre Diversidades, No. 8, enero-junio, pp. 15-52.

Tussie, D. (2014). Reshaping Regionalism and Regional Cooperation in South America. En Pensamiento Propio, No. 39, Cries, pp. 109-136.

Tussie, D. (2013). El estudio de la economía política internacional. En Legles, T.; Santa Cruz, A. y Zamudio, G. L., Introducción a las relaciones internacionales: América Latina y política global. Ciudad de México: Oxford University Press, pp. 51-61.

Vivares, E.; Lombardo, P. y Cvetich, K. (2013). Enfoques y cárceles conceptuales en el entendimiento de los nuevos regionalismos latinoamericanos. En Bonilla S., A. (Edit.), Desafios estratégicos del regionalismo contemporáneo Celac e Iberoamérica. San José Costa Rica: Flacso, pp. 21-46.

Vivares, E. y Dolcetti M., M. (2016). Regionalismos e integración en América Latina: situación actual y perspectivas. En Ayllón, B., ¿Latinoamérica dividida? Procesos de integración y cooperación Sur-Sur. Quito: Editorial Iaen, pp. 23-49.

Segib. (2014). Informe de la Cooperación Sur-Sur en Iberoamérica 2013-2014. Secretaria General Iberoamericana (Segib): Uruguay.

\section{D) DOCUMENTOS EN INTERNET}

Agencia Mexicana de Cooperación para el Desarrollo (Amexcid), (2018). Cooperación para el Desarrollo con las regiones prioritarias: Centroamérica y el Caribe 2012-2018. Libro Blanco. Disponible en: https://www.gob.mx/amexcid/ acciones-y-programas/cooperacion-de-mexicocon-centroamerica

Agenda Mesoamericana de Cooperación. Disponible en http://www.proyectomesoamerica.org/index. $\mathrm{php} /$ comunicacion/kit-de-comunicaciones-pm/ kit-tuxtla-2019

CSUCA. (2017). Estatuto del Consejo Superior Universitario Centroamericano. Guatemala: csucA. Disponible en http://www.csuca.org/docs-csuca/ documentosbasicos/Estatutocsuca2017.pdf

Declaración Conjunta de la Cumbre Extraordinaria de los Países Integrantes del Mecanismos de Diálogo y Concertación de Tuxtla. (2001). Disponible en http://www.minex.gob.gt/MDAa/ DATA/MDAA/20141125155847691CUMBRE\%20 EXTRAORDINARIA\%20DEL\%20MECANISMO $\% 20$ DE\%20DIALOGO\%20Y\%20CONCERTACION\%20 DE\%20TUXTLA \%2015\%20de\%20junio\%20 de\%202001\%20El\%20Salvador.pdf

Cumbres de Jefes de Estado y de Gobierno del Mecanismo de Diálogo y Concertación de Tuxtla (2008-2017).

Proyecto Mesoamérica. (s.f.). Proyecto de Integración y Desarrollo de Mesoamérica, años 2008-2015. Disponible en http://www.cepredenac.org/application/files/6014/8051/9734/Resumen_Ejecutivo.pdf 